\title{
Measuring Technology for a Magnetostrictive Microactuator
}

\author{
Albach, Thorsten; Sutor, Alexander; Lerch, Reinhard \\ Universität Erlangen-Nürnberg, Lehrstuhl für Sensorik \\ Paul-Gordan-Str. 3-5, 91052 Erlangen
}

\section{Introduction}

The use of materials which convert electrical into mechanical energy is a promising way to design new microsystems. Piezoelectric, magnetostrictive and shape memory materials are used here. Comparing the different conversion principles, magnetostrictive materials show high energy density, short reaction times and non-contact operation [1]. Research on these topics have been made at the Department of Sensor Technology at the University Erlangen-Nuremberg [2], [3], [4]. We fabricate long, narrow bending cantilevers on silicon substrate. Those cantilevers consist of passive and one active, magnetostrictive layer. If a magnetic field is applied along the cantilevers, they bend downwards. As a magnetostrictive material we use $\mathrm{Fe}_{49} \mathrm{Co}_{49} \mathrm{~V}_{2}$ (Vanadium Permendur). The fabrication of the microcantilevers has yet been subject to [4].

Here, we want to discuss the measuring technology we developed in order to measure the different properties of the microactuators. These are for example the direction of the magnetic Easy Axis and the saturation magnetostriction.

\subsection{Magnetostriction}

In $1842 \mathrm{~J}$. P. Joule discovered that iron changes in length, when a magnetic field is applied to it. This effect is now called the "Joule Magnetostriction" as a synonym for "magnetically induced strain". Also other ferromagnetic materials such as nickel, cobalt, some rare earths and alloys of them show this effect. The magnitude of the effect is described by the linear magnetostriction

$$
\lambda=\Delta L / L_{0}
$$

Material volume is considered constant. Figure 1 shows a simplified model of a magnetostrictive material. Above Curie temperature $T_{c}$, the magnetic domains are circular. Below Curie temperature they become oval and the material stretches by $\lambda_{0}$. If we apply a magnetic field $H_{\text {ext }}$ the magnetic domains rotate until saturation is reached at $H_{e x t}=H_{s}$. The corresponding magnetostriction is the so called saturation magnetostriction $\lambda_{s}$. The change in length is independent of the sign of $H_{\text {ext }}$.

\section{Fabrication}

We fabricate a magnetostrictive microactuator in the form of a bending cantilever on silicon substrate using MEMS-technology. The cantilever consists of layered thin films. Figure 2 shows a cross section of the device. We use $\mathrm{SiO}_{2}$ as passive layer and $\mathrm{Fe}_{49} \mathrm{Co}_{49} \mathrm{~V}_{2}$ as active layer on top of it. In between there is a thin $\mathrm{Cr}$ adhesion layer. The $\mathrm{SiO}_{2}$ is wet oxide, $\mathrm{Cr}$ and $\mathrm{Fe}_{49} \mathrm{Co}_{49} \mathrm{~V}_{2}$ are magnetron-HF-sputtered. The structure is then mechanically freed by anisotropic $\mathrm{KOH}$-etching. The cantilever measures $800 \mu \mathrm{m}$ in length, $75 \mu \mathrm{m}$ in width and its thickness amounts to $1 \mu \mathrm{m}$. If an external magnetic field is applied along the structure, the uppermost layer elongates and the whole device bends downwards. Maximum magnetostrictive strain of the active thin film can be achieved, when the magnetic easy axis lies in plane of the thin film and perpendicular to the external magnetic field [1]. In plane orientation of the easy axis is controlled through mechanical stress inside the layer. Materials with positive magnetostriction such as $\mathrm{Fe}_{49} \mathrm{Co}_{49} \mathrm{~V}_{2}$ show an 


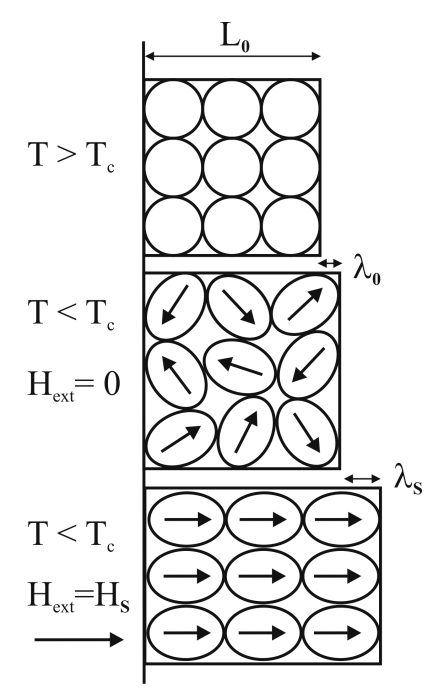

Figure 1: Joule magnetostriction caused by magnetic field $H_{e x t}$

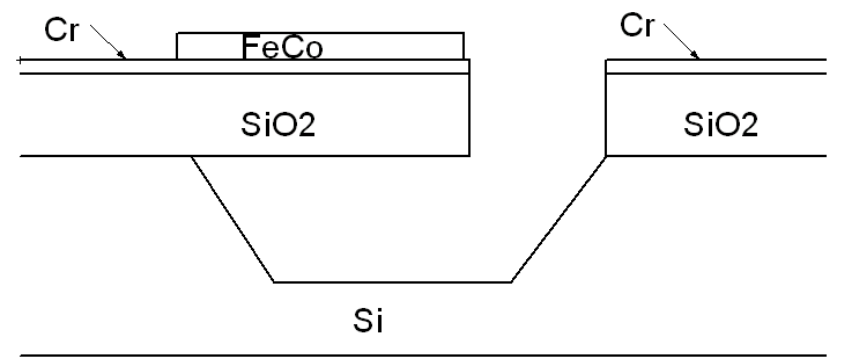

Figure 2: Cross section of the microactuator

in plane easy axis under tensile stress. For materials with negative magnetostriction this occurs under compressive stress. Since the sputtered metal $\mathrm{Fe}_{49} \mathrm{Co}_{49} \mathrm{~V}_{2}$ shows tensile stress, we assume to have an in plane orientation of the easy axis. Its direction inside the layer can be defined using an offset DC magnetic field during the sputtering process [5].

To apply a homogeneous DC-Offset magnetic field parallel to the substrate surface we use a field coil with pole shoes shown in figure 3. The silicon wafer is positioned on top of it. The whole assembly is mounted inside our sputtering unit. The applied flux density of up to $20 \mathrm{mT}$ does not affect the sputtering process in a remarkable manner.

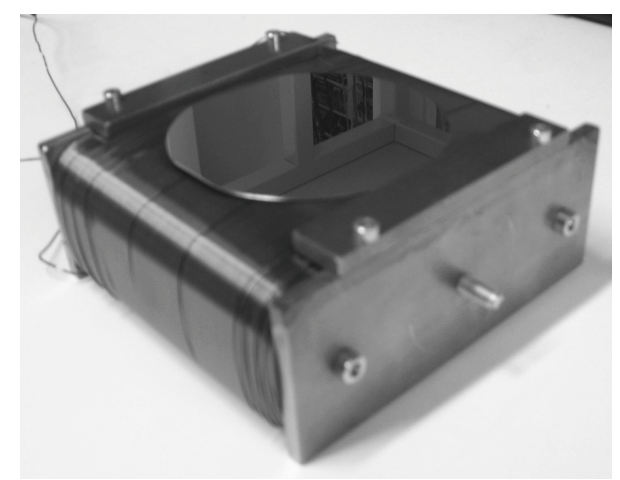

Figure 3: Field coil for DC offset field with Si wafer on top

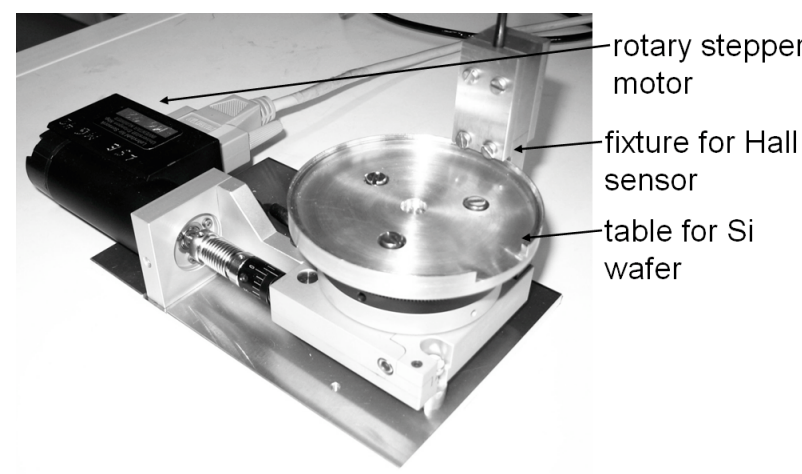

Figure 4: Measurement setup for measuring the direction of the easy axis

\section{Measuring Technology}

\subsection{Magnetic Easy Axis}

We use a measurement setup as depicted in figure 4 to measure the direction of the magnetization inside the magnetostrictive layer. We assume the direction of the in plane magnetic easy axis in this layer to have the same orientation as the measured magnetization. 
The silicon wafer is put on a table which is rotated by a precision rotary stepper motor. A Hall sensor is mounted in a defined distance and measures the remanent in plane flux density of the magnetostrictive layer in radial direction (figure 5). The whole setup is software controlled. The wafer is rotated past the sensor and for each angle we measure the radial flux density.

Figure 6 depicts a measurement for a $200 \mathrm{~nm}$ thick $\mathrm{Fe}_{49} \mathrm{Co}_{49} \mathrm{~V}_{2}$ film on silicon substrate. We applied a directional magnetic field of $B_{e x t}=22 \mathrm{mT}$ parallel to the substrate surface during the sputtering process. A distinct orientation of the magnetization of the film in the direction of the external magnetic field is visible. At the positions of the two wafer flats at angles of $0^{\circ}$ and $270^{\circ}$ we see a decrease in magnitude of the measured flux density. This happens due to the increased distance between the wafer and the Hall sensor. From this measurement we can assume the orientation of the magnetic easy axis of the $\mathrm{Fe}_{49} \mathrm{Co}_{49} \mathrm{~V}_{2}$ thin film to be in the direction of the magnetic field $B_{e x t}$ that we applied during sputtering.

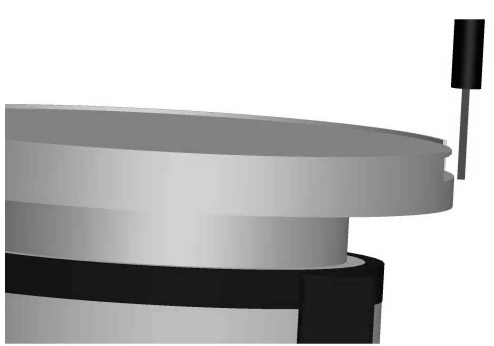

Figure 5: Position of Hall sensor for measuring radial flux density

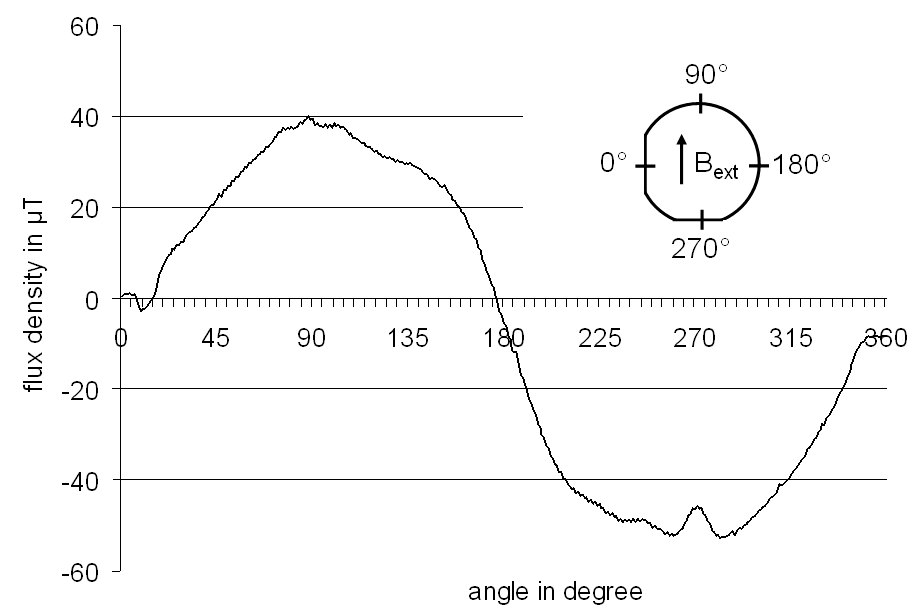

Figure 6: Measured remanent flux density in radial direction

\subsection{Measuring Magnetostriction}

Saturation magnetostriction $\lambda_{s}$ of the active thin film can be determined from the amount of bending of the cantilever. In [6], [7] we find a solution for $\lambda_{s}$ using a long and thin cantilever that is clamped at one end. Given an in plane easy axis, the calculation can be performed independent of the original direction of magnetization. We have to measure the tip deflections while applying a magnetic field parallel to the long side of the cantilever $\left(\Delta_{\|}\right)$and applying a field perpendicular to the long side $\left(\Delta_{\perp}\right)$. The material has to be driven into saturation in each direction. Saturation magnetostriction can then be calculated from the difference of both deflections as

$$
\lambda_{s}=-\frac{2}{9} \frac{\left(\Delta_{\|}-\Delta_{\perp}\right) h_{s}}{(1+\zeta) l^{2}}\left(\frac{1}{\zeta \chi}+4\left(1+\zeta^{2}\right)+\chi \zeta^{3}+6 \zeta\right),
$$

where

$$
\chi=\frac{\hat{Y}_{f}}{\hat{Y}_{s}} \quad \text { and } \quad \zeta=\frac{h_{f}}{h_{s}} .
$$

$\hat{Y}$ denotes the modulus of elasticity and $h$ denotes the thickness of the layers. The Index.$_{f}$ identifies the magnetostrictive film, the index . identifies the substrate. The length of the cantilever is $l$.

An external magnetic field is necessary to actuate the cantilever. The measuring station shown in figure 7 uses a field coil to induce a magnetic field inside a ferrite-yoke. The nearly homogeneous field in the centre over the air gap is used to actuate the cantilever in a defined way. The mechanical deflection of the cantilever is measured from above using a laser-scanning-vibrometer. The magnetic flux density is captured 
with a teslameter. The advantage of this assembly is the easy regulation of the magnetic flux density inside the air gap by adjusting the current in the coil. However, a big disadvantage lies in the ferrite-yoke itself. It shows magnetostrictively excited vibrations. We mount the laser-vibrometer and the fixture for the silicon wafer on a vibration damped table to avoid mechanical coupling between them and the ferrite-yoke.

Figure 8 shows the measured deflection of the cantilever tip vs. field strength at a frequency of $105 \mathrm{~Hz}$. We see the nonlinear curve which is typical for Joule magnetostriction. Saturation is reached at about $35 \mathrm{kA} / \mathrm{m}$. There is no more elongation of the $\mathrm{Fe}_{49} \mathrm{Co}_{49} \mathrm{~V}_{2}$ thin film at higher field strengths. The magnetostrictive elongation is independent of the sign of the applied magnetic field. Because of this the cantilever moves at twice the frequency of the driving field. There is also an overlayed cantilever movement with once the driving frequency which is generated by magnetic force. But this amplitude is small in comparison to the magnetostrictively excited one as can also be seen in figure 8 .

We drive the magnetostrictive layer into saturation at $35 \mathrm{kA} / \mathrm{m}$ and measure the deflection of the tip with a magnetic field applied parallel and perpendicular to the length of the cantilever. We get $\Delta_{\|}=11.4 \mu \mathrm{m}$ and $\Delta_{\perp}=1.1 \mu \mathrm{m}$. Using equation (2) we get the saturation magnetostriction of $\mathrm{Fe}_{49} \mathrm{Co}_{49} \mathrm{~V}_{2}$ as $\lambda_{s}=52 \cdot 10^{-6}$. In literature we can find $\lambda_{s}=67 \cdot 10^{-6}$ for a slightly different material $\left(\mathrm{Fe}_{50} \mathrm{Co}_{50}\right)$ [8].

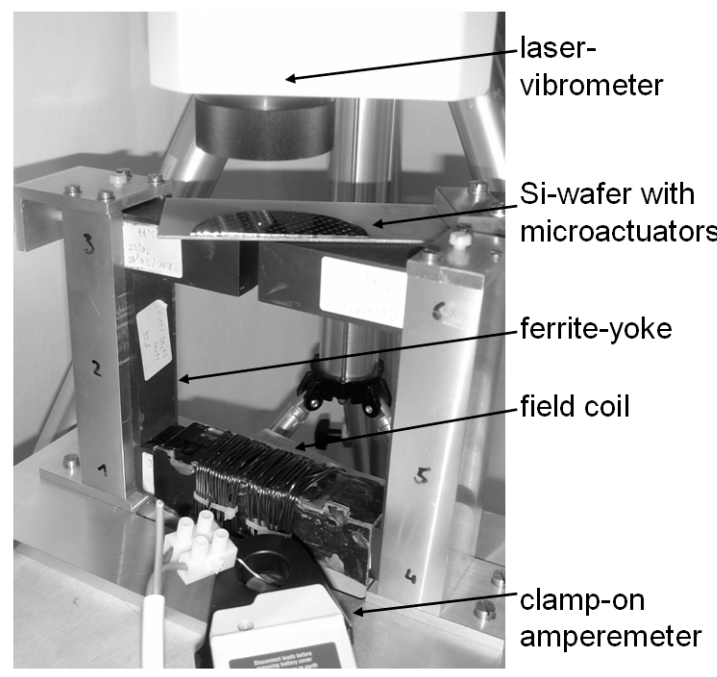

Figure 7: Measuring station for cantilever deflection using external magnetic excitation

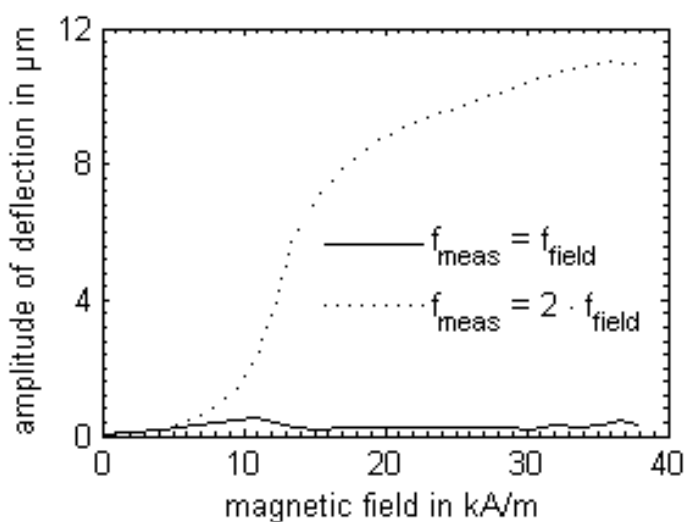

Figure 8: Deflection of cantilever tip vs. field strength

\section{Conclusion}

We reported the design and fabrication method of a magnetostrictive microactuator in form of a bending cantilever. To achieve a maximum tip deflection it is necessary to adjust the magnetic easy axis of the magnetostrictive film to be perpendicular to the driving magnetic field. We do this during thin film deposition by applying a DC offset magnetic field. Our measurement method shows the magnetic easy axis to be aligned with the direction of this field.

To excite movement of our micro cantilevers we use a measurement setup with a field coil on a ferrite yoke. The tip deflection of the cantilevers is measured from above by a laser vibrometer. From the tip deflection at magnetic saturation we can calculate the saturation magnetostriction of our active material $\mathrm{Fe}_{49} \mathrm{Co}_{49} \mathrm{~V}_{2}$ to be $\lambda_{s}=52 \cdot 10^{-6}$. 


\section{References}

[1] Quandt, E.: Giant Magnetostrictive Thin Film Technologies in Engdal, G.: Handbook of Giant Magnetostrictive Materials, Academic Press, 2000

[2] Baffoun, B., Lerch, R. Bimorph magnetostrictive microactuator based on silicon bulk-micromachining, Proc. 9th Int. Conf. New Actuators, ACTUATOR 2004, pp. 577-580

[3] Baffoun, B., Sutor, A., Lerch, R. Development in Combined Si-based Magnetic Microactuator, Proc. IEEE Sensors 2005, Irvine, USA

[4] Albach, T., Baffoun, B., Sutor, A., Lerch, R., Entwicklung eines magnetostriktiven Mikroaktors, Mikrosystemtechnik-Kongress 2007, Dresden

[5] Quandt, E.: Giant magnetostrictive thin film materials and applications, J. Alloys Comp. 258 (1997) pp. 126-132

[6] Victor H.Guerrero, Robert C.Wetherhold, Magnetostrictive bending of cantilever beams and plates, J. of Applied Physics, 2003, pp 6659-6666, Vol. 294, No. 10

[7] Gehring, G. A., Cooke, M. D., Gregory, I. S. , Karl, W. J. and Watts R., Cantilever unified theory and optimization for sensors and actuators, Smart Mater. Struct. 9 (2000) pp. 918-931

[8] Bozorth, R. M., Ferromagnetism, IEEE Press, Piscataway, NJ, 1993 